\title{
Usefulness of noninvasive biomarkers of fibrosis in chronic liver disease
}

Disease etiology is a major factor in the performance of noninvasive fibrosis biomarkers, according to new research published in Alimentary Pharmacology \& Therapeutics. Moreover, the findings from this large-scale study indicate that APRI (aspartate aminotransferase:platelet ratio index) and Fibrotest (BioPredictive, Paris, France) perform best for the diagnosis of marked fibrosis in patients with chronic liver disease (CLD).

Sebastiani and colleagues directly compared the performance of several validated biomarkers-FibroTest, APRI and Forn's index, among others-with that of liver biopsy (the gold standard) for the diagnosis of liver fibrosis in 2,411 patients who had CLDs of different etiologies (including nonalcoholic steatohepatitis or infection with $\mathrm{HCV}$ ).

Overall, diagnostic performance was highest for APRI and Fibrotest compared with the other biomarkers assessed.

Moreover, the researchers found that the major factor influencing the performance of the biomarkers was the etiology of disease, and that the stage of fibrosis could also affect the diagnostic efficiency of biomarkers, especially in patients with a peculiar distribution of fibrosis.

"Staging of liver fibrosis [is] ... of paramount importance for both prognosis and management of CLDs," write the authors. The research team note that, in those with marked fibrosis, liver biopsy should still be the first-choice diagnostic procedure and that more research is needed before the application of noninvasive biomarkers for the diagnosis of liver fibrosis in all disease etiologies becomes standard.

\section{Paola Quattroni}

Original article Sebastiani, G. et al. The impact of liver disease aetiology and the stage of hepatic fibrosis on the performance of non-invasive fibrosis biomarkers: an internal study of 2411 cases. Aliment. Pharmacol. Ther. 34, 1202-1216 (2011) 